\title{
Semiclassical matrix model for quantum chaotic transport with time-reversal symmetry
}

\author{
Marcel Novaes \\ Instituto de Física, Universidade Federal de Uberlândia, Uberlândia, MG, \\ 38408-100, Brazil \\ E-mail: marcel.novaes@gmail.com
}

\begin{abstract}
We show that the semiclassical approach to chaotic quantum transport in the presence of time-reversal symmetry can be described by a matrix model, i.e. a matrix integral whose perturbative expansion satisfies the semiclassical diagrammatic rules for the calculation of transport statistics. This approach leads very naturally to the semiclassical derivation of universal predictions from random matrix theory.

PACS numbers: 05.45.Mt,03.65.Sq,73.23.Ad
\end{abstract}

\section{Introduction}

We consider wave scattering through a system in which the corresponding ray dynamics is strongly chaotic. Under a minimal information statistical approach, it is natural to model the scattering $\mathcal{S}$ matrix as a random matrix, which must be unitary by conservation principles [1, 2, 3]. The quantity $\mathcal{S}_{i j}$ is the scattering amplitude from channel $j$ to channel $i$. In the presence of time-reversal symmetry, scattering from $i$ to $j$ is equivalent to scattering from $j$ to $i$ and so $\mathcal{S}$ must also be symmetric. The ensemble of unitary symmetric complex matrices has a natural probability measure on it, and is known and the Circular Orthogonal Ensemble (COE) of random matrix theory (RMT).

RMT predicts successfully several scattering observables, in agreement with numerical simulations and experimental results, such as average conductance, conductance fluctuations, average shot-noise and higher counting statistics. This approach has been reviewed in [4, and some recent results include [5, 6, 7, 8, 9, 10, It is rather flexible, and can be adapted in order to treat the statistics of time delay [11, 12, 13, and to consider the presence of superconductors [15, 16, non-ideal contacts [17, 18, 19, 20, 21], graphene [22, 23], etc.

Recovering RMT results from chaotic scattering trajectories has long been a central problem for the semiclassical approach to quantum mechanics, in which $\mathcal{S}_{i j}$ is expressed as a sum over paths leading from $j$ to $i$ [24, 25]. In order to reproduce quantum effects, it is necessary to consider trajectories that are action-correlated on the scale of $\hbar$. This started to be done perturbatively in [26, 27] and was shown to give the exact result for the simplest observables in [28, 29, 30]. Attention then turned to more general transport statistics [31, 32, 33, 34, until complete equivalence was shown 
between semiclassics and RMT [35, 36] (in the meantime, semiclassics was able to go beyond RMT, incorporating effects due to finite Ehrenfest time, see e.g. [37, 38, 39]).

These semiclassical works rely on elaborate manipulations with diagrams and/or permutations. A more direct demonstration of the RMT-semiclassics equivalence was presented in [40, based on a matrix model formulation of the semiclassical approximation. This is a matrix integral whose diagrammatic expansion satisfies exactly the same diagrammatic rules as the semiclassical calculation of transport observables, and which turns out to be equivalent to usual RMT.

However, the treatment in [40] concerns only systems where time-reversal symmetry is broken. The purpose of this work is to extend the matrix model approach to time-reversal symmetric systems, establishing the RMT-semiclassics equivalence in a direct way for this universality class.

\section{Usual RMT treatment}

Suppose a chaotic cavity, coupled to two ideal leads supporting $N_{1}$ and $N_{2}$ open channels, having a $M$-dimensional $\mathcal{S}$ matrix where $M=\left(N_{1}+N_{2}\right)$. The energy of the incoming wave is $E$, and the classical dynamics in the cavity is assumed fully chaotic at this energy. Under this assumption, RMT assumes the statistical properties of transport to be independent of $E$.

We shall consider the quantities

$$
P(\vec{i}, \vec{j})=\mathcal{S}_{i_{1} i_{2}} \mathcal{S}_{i_{3} i_{4}} \cdots \mathcal{S}_{i_{2 n-1} i_{2 n}} \mathcal{S}_{j_{1} j_{2}}^{*} \mathcal{S}_{j_{3} j_{4}}^{*} \cdots \mathcal{S}_{j_{2 n-1} j_{2 n}}^{*},
$$

which can be used to expand any generic observable (all matrices are taken at the same energy). For instance, the (dimensionless) conductance and shot-noise are given by

$$
g=\sum_{i_{1}, i_{2}} \mathcal{S}_{i_{1} i_{2}} \mathcal{S}_{i_{1} i_{2}}^{*}, \quad p=g-\sum_{i_{1}, i_{2}, i_{3}, i_{4}} \mathcal{S}_{i_{1} i_{2}} \mathcal{S}_{i_{3} i_{2}}^{*} \mathcal{S}_{i_{3} i_{4}} \mathcal{S}_{i_{1} i_{4}}^{*},
$$

where the sums over $i_{2}, i_{4}\left(i_{1}, i_{3}\right)$ run over the $N_{1}$ incoming ( $N_{2}$ outgoing) channels.

According to the random matrix theory approach, for time-reversal invariant chaotic systems $\mathcal{S}$ is uniformly distributed in the $\operatorname{COE}(M)$. The interest then lies in the average value of (1) over this ensemble. The simplest case is

$$
\left\langle\left|\mathcal{S}_{i j}\right|^{2}\right\rangle_{\mathrm{COE}(\mathrm{M})}=\frac{1+\delta_{i j}}{M+1}
$$

which leads to the well known prediction $N_{1} N_{2} /(M+1)$ for the average conductance. In general, the average vanishes unless $\vec{j}$ is equal to some permutation of $\vec{i}$. For instance,

$$
\left\langle\mathcal{S}_{12} \mathcal{S}_{12}^{*} \mathcal{S}_{34} \mathcal{S}_{34}^{*}\right\rangle_{\mathrm{COE}(\mathrm{M})}=\frac{M+2}{M(M+1)(M+3)},
$$

and

$$
\left\langle\mathcal{S}_{12} \mathcal{S}_{14}^{*} \mathcal{S}_{34} \mathcal{S}_{23}^{*}\right\rangle_{\mathrm{COE}(\mathrm{M})}=\frac{-1}{M(M+1)(M+3)} .
$$

There may be several possibilities for the permutation relating $\vec{j}$ and $\vec{i}$, and the general expression for our average contains a sum,

$$
\langle P(\vec{i}, \vec{j})\rangle_{\mathrm{COE}(\mathrm{M})}=\sum_{\pi \in \mathfrak{S}_{2 n}} \delta_{\pi}(\vec{i}, \vec{j}) \mathrm{W}_{M}(\pi)
$$


where $\mathfrak{S}_{n}$ is the permutation group of $n$ symbols and

$$
\delta_{\pi}(\vec{i}, \vec{j})=\prod_{k=1}^{2 n} \delta_{i_{k}, j_{\pi(k)}} .
$$

The quantity $\mathrm{W}_{M}(\pi)$, called the Weingarten function of the COE, can be computed from explicit character-theoretic formulas [4] or via recurrence relations [42.

Since the $\mathcal{S}$-matrix is symmetric, the value of (6) must be invariant under the action of any permutation that interchanges $j_{2 k-1}$ with $j_{2 k}$, or any permutation that interchanges simultaneously $j_{2 k-1}$ with $j_{2 r-1}$ and $j_{2 k}$ with $j_{2 r}$. The set of all such permutations is called the hyperoctahedral group $\mathfrak{H}_{n} \subset \mathfrak{S}_{2 n}$. For $n=2$ it consists of the permutations $\{1,(12),(34),(12)(34)\}$. In general, $\mathfrak{H}_{n}$ has $2^{n} n$ ! elements. Thus, if $\xi \in \mathfrak{H}_{n}$ then

$$
\mathrm{W}_{M}(\pi \xi)=\mathrm{W}_{M}(\pi)
$$

\section{Semiclassical Diagrammatics}

The semiclassical approximation to $\mathcal{S}_{i j}$ requires trajectories starting at channel $j$ and ending at channel $i$. In the semiclassical expression for (1) we end up with some trajectories (direct ones) going from $i_{2 k}$ to $i_{2 k-1}$ and some other trajectories (partner ones) going from $j_{2 k}$ to $j_{2 k-1}$. Consider now the average value of (1) over a certain energy window, $\langle P(\vec{i}, \vec{j})\rangle_{E}$, this window being small in the classical scale but large in the quantum scale; as $\hbar \rightarrow 0$, constructive interference is required and the result is determined by correlations: partner trajectories must have almost the same collective action as direct ones.

The theory of correlated chaotic trajectories has been discussed in detail in a number of papers [28, 29, 30, 32, 36]. Trajectories from correlated sets may differ only in small regions (called encounters) in which the direct ones run nearly parallel or anti-parallel, while the partner ones have crossings. This ensures they have almost the same collective action. In particular, this implies that $\vec{i}$ and $\vec{j}$ must be equal up to a permutation, a condition already met in the RMT treatment.

These trajectory multiplets are usually represented by diagrams, in which the complicated pieces of chaotic trajectories in-between encounters are depicted as simple links. Calculation of any given $\langle P(\vec{i}, \vec{j})\rangle_{E}$ requires constructing all possible contributing diagrams. Most importantly, a diagrammatic rule has been devised for the value of any diagram: it is $(-1)^{V} M^{V-L}$, where $V$ is the number of encounters and $L$ is the number of links.

We show two examples in Figure 1. Panel a) shows a contribution to $\mathcal{S}_{i_{1} i_{2}} \mathcal{S}_{i_{3} i_{2}}^{*} \mathcal{S}_{i_{3} i_{4}} \mathcal{S}_{i_{1} i_{4}}^{*}$, containing two 2-encounters. One of the encounters involves two different trajectories, while the other one is of a trajectory with itself. Notice how the direct and partner trajectories run in opposite senses in one of the regions. This contribution is only possible because we are assuming the system has time-reversal symmetry. Its value is $1 / M^{4}$. Panel b) shows a contribution to $\mathcal{S}_{i_{1} i_{2}} \mathcal{S}_{i_{3} i_{2}}^{*} \mathcal{S}_{i_{3} i_{4}} \mathcal{S}_{i_{5} i_{4}}^{*} \mathcal{S}_{i_{5} i_{6}} \mathcal{S}_{i_{1} i_{6}}^{*}$, containing a single 3 -encounter. This diagram does not require time-reversal symmetry, and its value is $-1 / M^{5}$.

A different diagrammatic representation, more convenient, of correlated trajectories uses ribbon graphs [36. In this case we turn every encounter into a vertex, and trajectories are depicted as edges of ribbons. It is sometimes necessary to perform twists on some of the ribbons. The diagrams from Figure 1a,b are represented as ribbon graphs in Figure 2a,b. 
a)

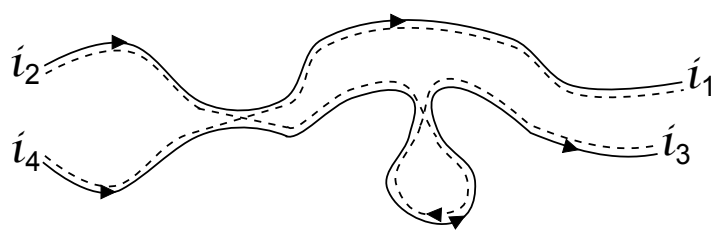

b)

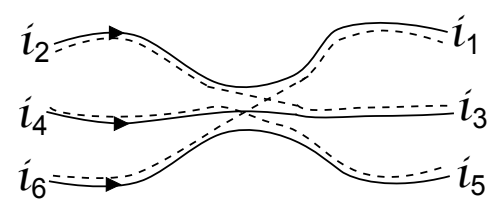

Figure 1. Two examples of semiclassical diagrams required in the calculation of transport observables. Direct trajectories (solid lines) are correlated with partner trajectories (dashed lines), by means of 2-encounters (left) and a 3-encounter (right). This is very simplified: actual trajectories are long and chaotic.
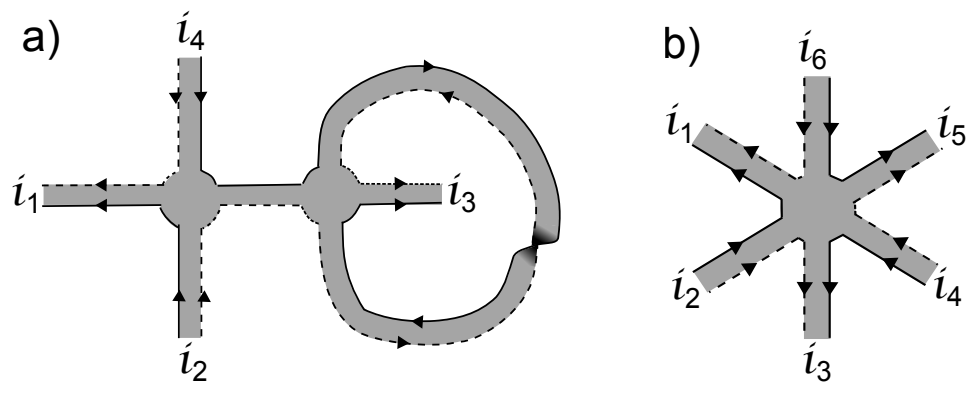

Figure 2. A different representation of the semiclassical diagrams of Figure 1, in terms of ribbon graphs. Encounters become vertices and trajectories become edges of ribbons. Notice how one of the ribbons must be twisted in the first case.

\section{Matrix model for the semiclassical approach}

\subsection{Wick's rule}

Suppose the matrix integral

$$
\langle f(S)\rangle_{S}=\frac{1}{\mathcal{Z}} \int d S e^{-\frac{M}{2} \operatorname{Tr} S S^{T}} f(S),
$$

where $M$ is a parameter and

$$
\mathcal{Z}=\int d S e^{-\frac{M}{2} \operatorname{Tr} S S^{T}}
$$

is a normalization factor. This integral runs over general $N$-dimensional real matrices, without constraints (this is known as the real Ginibre ensemble [43]). Notice that the dimension $N$ is not related to channel numbers; we continue with $M=N_{1}+N_{2}$.

Gaussian integrals can be performed exactly, leading to

$$
\left\langle S_{a b} S_{c d}\right\rangle_{S}=\frac{\delta_{a c} \delta_{b d}}{M} .
$$

When there are many matrix elements being integrated (the number must be even, otherwise the result vanishes), we may use the well known Wick's rule,

$$
\left\langle\prod_{k=1}^{2 n} S_{a_{k} b_{k}}\right\rangle_{S}=\sum_{\sigma \in \mathfrak{M}_{n}} \prod_{k=1}^{n}\left\langle S_{a_{\sigma(2 k-1)} b_{\sigma(2 k-1)}} S_{a_{\sigma(2 k)} b_{\sigma(2 k)}}\right\rangle_{S}
$$


Semiclassical matrix model for quantum chaotic transport with time-reversal symmetry 5
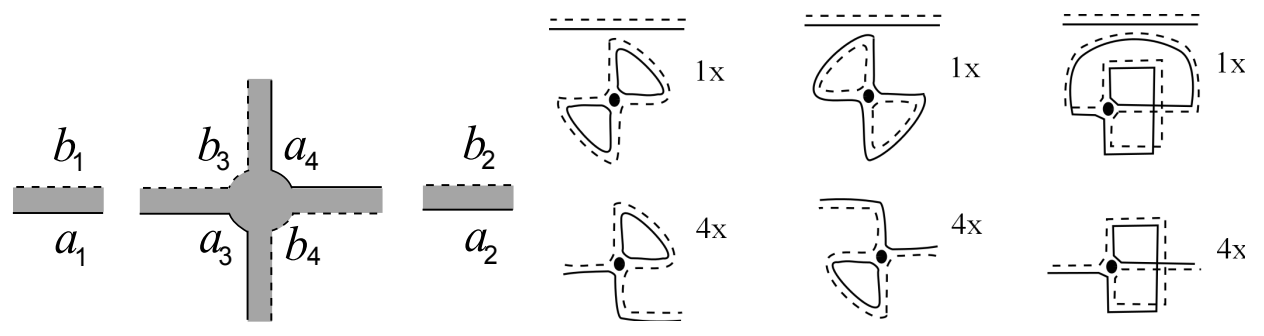

Figure 3. Diagrammatical representation of the average value of $\operatorname{Tr}\left(S S^{T}\right)^{2} S_{a_{1} b_{1}} S_{a_{2} b_{2}}$, and the possible connections between lines, according to Wick's rule. In the upper line a direct connection is made between $S_{a_{1} b_{1}}$ and $S_{a_{2} b_{2}}$. There are in total 15 connections, and we show how many times each topology appears, due to trivial symmetries.

where the sum is over all possible matchings among $2 n$ elements. For example, at $n=2$ there are three possible matchings,

$$
\begin{aligned}
& \left\langle S_{a_{1} b_{1}} S_{a_{2} b_{2}} S_{a_{3} b_{3}} S_{a_{4} b_{4}}\right\rangle=\left\langle S_{a_{1} b_{1}} S_{a_{2} b_{2}}\right\rangle\left\langle S_{a_{3} b_{3}} S_{a_{4} b_{4}}\right\rangle \\
& +\left\langle S_{a_{1} b_{1}} S_{a_{3} b_{3}}\right\rangle\left\langle S_{a_{2} b_{2}} S_{a_{4} b_{4}}\right\rangle+\left\langle S_{a_{1} b_{1}} S_{a_{4} b_{4}}\right\rangle\left\langle S_{a_{2} b_{2}} S_{a_{3} b_{3}}\right\rangle .
\end{aligned}
$$

Elements of $\mathfrak{M}_{n}$ can be represented by permutations acting on the trivial matching $\{\{1,2\},\{3,4\}, \ldots\}$. In this sense, the above matchings correspond to the identity permutation, to the transposition (23) and to the cycle (243). A permutation $\sigma$ represents a matching if and only if it satisfies $\sigma(2 i-1)<\sigma(2 i)$ and $\sigma(2 i-1)<$ $\sigma(2 i+1)$. Any element of $\mathfrak{S}_{2 n}$ can be decomposed uniquely as the product of a member of $\mathfrak{M}_{n}$ and a member of $\mathfrak{H}_{n}$, i.e. the set $\mathfrak{M}_{n}$ can be seen as the coset $\mathfrak{S}_{2 n} / \mathfrak{H}_{n}$.

\subsection{Diagrammatics}

Introduce the following diagrammatical representation to the calculation of (12). Each matrix element $S_{a b}$ is represented by a ribbon, having one edge associated with $a$ and depicted with a solid line, and the other edge associated with $b$ and depicted with a dashed line. Wick's rule then tells us to draw all possible connections among these lines, and associate to each connection a factor $1 / M$. When two ribbons are connected, their solid edges merge and must have the same label, and likewise for the dashed edges.

For example, consider the average value

$$
\left\langle\operatorname{Tr}\left(S S^{T}\right)^{2} S_{a_{1} b_{1}} S_{a_{2} b_{2}}\right\rangle=\sum_{a_{3} a_{4} b_{3} b_{4}=1}^{N}\left\langle S_{a_{1} b_{1}} S_{a_{2} b_{2}} S_{a_{3} b_{3}} S_{a_{4} b_{3}} S_{a_{4} b_{4}} S_{a_{3} b_{4}}\right\rangle .
$$

The presence of the trace requires repeated indices among the matrix elements. This is taken into account by arranging them around a vertex. We show in Figure 3 the ribbons associated with (14), and the possible connections in the application of Wick's rule. There are six distinct topologies, each appearing with some multiplicity. The calculation results in the value

$$
\frac{-1}{M^{3}}\left(2 N^{3}+N^{2}+8 N+4\right) \delta_{a_{1} a_{2}} \delta_{b_{1} b_{2}} .
$$

The power of $N$ counts the number of indices being freely summed over, associated with closed lines in the diagrams. 
This kind of diagrammatics has been extensively discussed in the literature. The most common situation is when complex hermitian matrices are used 44, in which case no twists are required in the ribbons (they are orientable) and no distinction is made between the edges. Complex non-hermitian matrices [45] maintain orientability but allow different edges. For real symmetric matrices the ribbons are not necessarily orientable [46, 47] and edges are indistinguishable. Our case (general real matrices) does not require orientability, and has distinguished edges.

\subsection{Introducing channels}

We are using the labels $a$ and $b$ to identify the 'trajectories' of our matrix model. We must still introduce the channel labels. To that end, let $Q=1_{M} \oplus 0_{N-M}$ be an orthogonal projector, having the $M$-dimensional identity in its upper-left corner and all other entries equal to zero. Let us also define the matrix

$$
R=X Q S Q X^{\dagger},
$$

where $X$ is some complex $M \times M$ matrix. This leads to

$$
\prod_{k=1}^{2 n} R_{i_{k} i_{k}}=\prod_{k=1}^{2 n} \sum_{a_{k}, b_{k}=1}^{M} X_{i_{k} a_{k}} S_{a_{k} b_{k}} X_{i_{k} b_{k}}^{*} .
$$

The indices $i_{k}$ represent the channels. The idea is that, upon taking the average over the $S$ matrices, the $a$-labels will produce the direct trajectories connecting the channels, while the $b$-labels will produce the partner trajectories, which may connect the channels in a different way but will necessarily be correlated with the direct ones.

For future reference, let us also define the matrix $Z=X X^{T}$. Notice that this is a complex symmetric matrix. The elements of $Z$ will be related to direct trajectories, and the elements of $Z^{*}$ will be related to the partner trajectories.

Before going into further details of this method, let us show it in action for the simplest example.

\subsection{The conductance}

Conductance requires the average value of $\mathcal{S}_{i_{1} i_{2}} \mathcal{S}_{i_{1} i_{2}}^{*}$. We start by writing

$$
\left\langle R_{i_{1} i_{1}} R_{i_{2} i_{2}}\right\rangle_{S}=\sum_{a_{1} a_{2} b_{1} b_{2}=1}^{M} X_{i_{1} a_{1}} X_{i_{2} a_{2}} X_{i_{1} b_{1}}^{*} X_{i_{2} b_{2}}^{*}\left\langle S_{a_{1} b_{1}} S_{a_{2} b_{2}}\right\rangle_{S}=\frac{1}{M} Z_{i_{1} i_{2}} Z_{i_{1} i_{2}}^{*} .
$$

The coefficient $1 / M$ is related to the leading order approximation to Eq.(3).

Next, we can produce diagrams with encounters by including traces of powers of $S S^{T}$. The simplest such case is

$$
\left\langle\frac{-M}{4} \operatorname{Tr}\left(S S^{T}\right)^{2} R_{i_{1} i_{1}} R_{i_{2} i_{2}}\right\rangle_{S}=\frac{-1}{4 M^{2}}\left(4+8 N+N^{2}+2 N^{3}\right) Z_{i_{1} i_{2}} Z_{i_{1} i_{2}}^{*} .
$$

The diagrammatics of this average is the same as the one shown in Figure 3. We have multiplied by $-M$ because we already know that each encounter must be accompanied by such factor in the semiclassical diagrammatics. We have divided by 4 because of the rotation symmetry of the 2-encounter.

As we have seen, closed lines produce powers of $N$ (the $S$-matrices inside the trace are not truncated). In a semiclassical interpretation, such closed lines would represent periodic orbits, forever trapped inside the system. The true semiclassical 
diagrams contain only scattering trajectories, and no periodic orbits. We can get rid of such orbits by the trick of letting $N \rightarrow 0$. If we perform this trick, we get $Z_{i_{1} i_{2}} Z_{i_{1} i_{2}}^{*}$ with a coefficient of $-1 / M^{2}$, which is the second order approximation to Eq.(13).

A triple encounter corresponds to

$$
\lim _{N \rightarrow 0}\left\langle\frac{-M}{6} \operatorname{Tr}\left(S S^{T}\right)^{3} R_{i_{1} i_{1}} R_{i_{2} i_{2}}\right\rangle_{S}=\frac{-1}{M^{3}} Z_{i_{1} i_{2}} Z_{i_{1} i_{2}}^{*},
$$

where we have discounted a 6 -fold rotation symmetry. The situation with two single encounters correspond to

$$
\lim _{N \rightarrow 0}\left\langle\frac{1}{2}\left[\frac{-M}{4} \operatorname{Tr}\left(S S^{T}\right)^{2}\right]^{2} R_{i_{1} i_{1}} R_{i_{2} i_{2}}\right\rangle_{S}=\frac{2}{M^{3}} Z_{i_{1} i_{2}} Z_{i_{1} i_{2}}^{*} .
$$

Here there is an extra denominator of 2 to account for the exchange symmetry between the encounters. The values of (20) and (21) together produce a coefficient to $Z_{i_{1} i_{2}} Z_{i_{1} i_{2}}^{*}$ which is $1 / M^{3}$, related precisely to the third order approximation to Eq.(3).

In order to produce all possible encounters, we must include all possible traces, each one of them multiplied by $-M$, as required by the semiclassical diagrammatic rules. We must also discount an overcounting of $2 q$ from rotation symmetry around vertices of valence $q$, and take into account the exchange symmetry between encounters of the same valence. Fortunately, all this is automatically implemented by means of an exponential function.

Therefore, in analogy to [40, our semiclassical matrix model for the average value of $\mathcal{S}_{i_{1} i_{2}} \mathcal{S}_{i_{1} i_{2}}^{*}$ is to compute

$$
\lim _{N \rightarrow 0}\left\langle e^{-M \sum_{q \geq 2} \frac{1}{2 q} \operatorname{Tr}\left[\left(S S^{T}\right)^{q}\right]} R_{i_{1} i_{1}} R_{i_{2} i_{2}}\right\rangle_{S},
$$

and to extract the coefficient of $Z_{i_{1} i_{2}} Z_{i_{1} i_{2}}^{*}$. In order to compute the conductance, we just sum over $i_{1}, i_{2}$.

\subsection{Higher Moments}

To treat conductance, which involves two channels, we considered the quantity $R_{i_{1} i_{1}} R_{i_{2} i_{2}}$. In order to be able to obtain a semiclassical matrix model for higher transport moments, involving $n$ channels, we must consider more general quantities of the form $\prod_{k=1}^{2 n} R_{i_{k} i_{k}}$.

As an example, let us look at the diagrammatics of this average value for $n=2$. Using Wick's rule, it is easy to see that this is given by

$$
\begin{aligned}
& \left\langle R_{i_{1} i_{1}} R_{i_{2} i_{2}} R_{i_{3} i_{3}} R_{i_{4} i_{4}}\right\rangle_{S}=\frac{1}{M^{2}}\left[Z_{i_{1} i_{2}} Z_{i_{1} i_{2}}^{*} Z_{i_{3} i_{4}} Z_{i_{3} i_{4}}^{*}\right. \\
& \left.+Z_{i_{1} i_{3}} Z_{i_{1} i_{3}}^{*} Z_{i_{2} i_{4}} Z_{i_{2} i_{4}}^{*}+Z_{i_{1} i_{4}} Z_{i_{1} i_{4}}^{*} Z_{i_{2} i_{3}} Z_{i_{2} i_{3}}^{*}\right] .
\end{aligned}
$$

The channel labels appear in different arrangements on the right hand side, corresponding to the semiclassical diagrams in Figure 4a. This quantity is thus producing all the leading order transport diagrams with four channels simultaneously.

If we wish to know the leading order approximation to the average value of a certain quantity, say $\mathcal{S}_{i_{1} i_{2}} \mathcal{S}_{j_{1} j_{2}}^{*} \mathcal{S}_{i_{3} i_{4}} \mathcal{S}_{j_{3} j_{4}}^{*}$, we just extract from the above result the coefficient of $\left[Z_{i_{1} i_{2}} Z_{j_{1} j_{2}}^{*} Z_{i_{3} i_{4}} Z_{j_{3} j_{4}}^{*}\right]$ (when performing this coefficient extraction procedure, we consider the indices to be independent variables, i.e. we do not care for possible coincidences among the elements of $\vec{i}$ ). We shall use the notation $[x] f$ to denote the coefficient of $x$ in $f$. 
a)

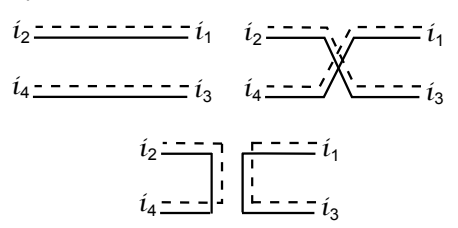

b)
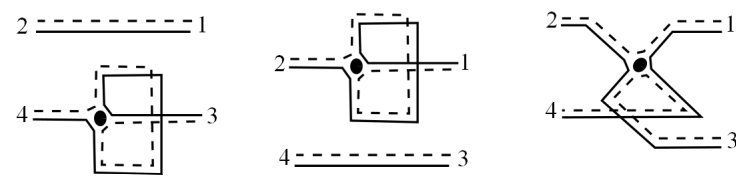

Figure 4. a) Diagrams related to the leading order approximation to $\left\langle R_{i_{1} i_{1}} R_{i_{2} i_{2}} R_{i_{3} i_{3}} R_{i_{4} i_{4}}\right\rangle_{S}$. b) The first two diagrams are responsible for Eq. 228, while the last diagram is responsible for Eq.(29).

For example, looking at Eq.(23) it is clear that

$$
\left[Z_{i_{1} i_{2}} Z_{i_{3} i_{4}}\right]\left\langle\prod_{k=1}^{4} R_{i_{k} i_{k}}\right\rangle_{S}=\frac{1}{M^{2}} Z_{i_{1} i_{2}}^{*} Z_{i_{3} i_{4}}^{*} .
$$

On the other hand, when we consider $\left[Z_{j_{1} j_{2}}^{*} Z_{j_{3} j_{4}}^{*}\right] Z_{i_{1} i_{2}}^{*} Z_{i_{3} i_{4}}^{*}$, it follows that $\vec{j}$ and $\vec{i}$ can only differ by a hyperoctahedral permutation: either we have the set identities $\left\{j_{1}, j_{2}\right\}=\left\{i_{1}, i_{2}\right\},\left\{j_{3}, j_{4}\right\}=\left\{i_{3}, i_{4}\right\}$ or the set identities $\left\{j_{1}, j_{2}\right\}=\left\{i_{3}, i_{4}\right\},\left\{j_{3}, j_{4}\right\}=$ $\left\{i_{1}, i_{2}\right\}$. That is, we have that

$$
\left[Z_{j_{1} j_{2}}^{*} Z_{j_{3} j_{4}}^{*}\right] Z_{i_{1} i_{2}}^{*} Z_{i_{3} i_{4}}^{*}=\sum_{\pi \in \mathfrak{H}_{2}} \delta_{\pi}(\vec{i}, \vec{j}) .
$$

In conclusion, our semiclassical prediction for the leading order approximation to the average value of $\mathcal{S}_{i_{1} i_{2}} \mathcal{S}_{j_{1} j_{2}}^{*} \mathcal{S}_{i_{3} i_{4}} \mathcal{S}_{j_{3} j_{4}}^{*}$ is $M^{-2} \sum_{\pi \in \mathfrak{H}_{2}} \delta_{\pi}(\vec{i}, \vec{j})$.

More concretely, we have, for instance,

$$
\left[Z_{12} Z_{12}^{*} Z_{34} Z_{34}^{*}\right]\left\langle R_{11} R_{22} R_{33} R_{44}\right\rangle_{S}=\frac{1}{M^{2}},
$$

which is indeed the leading order approximation to Eq.(4), and

$$
\left[Z_{12} Z_{14}^{*} Z_{34} Z_{23}^{*}\right]\left\langle R_{11} R_{22} R_{33} R_{44}\right\rangle_{S}=0,
$$

reflecting the fact that there are no semiclassical diagrams contributing at leading order to the calculation of Eq.(5), and that indeed Eq.(5) is of order $M^{-3}$.

We can produce perturbative corrections, i.e. diagrams with encounters, by including traces, as we did for the conductance. For instance, a single encounter is introduced as

$$
\lim _{N \rightarrow 0}\left[Z_{12} Z_{12}^{*} Z_{34} Z_{34}^{*}\right]\left\langle\frac{-M}{4} \operatorname{Tr}\left(S S^{T}\right)^{2} \prod_{k=1}^{4} R_{k k}\right\rangle_{S}=\frac{-2}{M^{3}},
$$

which is the first correction to Eq.(44), and

$$
\lim _{N \rightarrow 0}\left[Z_{12} Z_{14}^{*} Z_{34} Z_{23}^{*}\right]\left\langle\frac{-M}{4} \operatorname{Tr}\left(S S^{T}\right)^{2} \prod_{k=1}^{4} R_{k k}\right\rangle_{S}=\frac{-1}{M^{3}},
$$

which is the leading order contribution to Eq.(15). The diagrams corresponding to the above results can be seen in Figure 4b. As already discussed, the limit $N \rightarrow 0$ is necessary to discard diagrams having periodic orbits. 
Semiclassical matrix model for quantum chaotic transport with time-reversal symmetry 9

As we saw previously, in order to produce all possible encounters we must introduce the factor $e^{-M \sum_{q \geq 2} \frac{1}{2 q} \operatorname{Tr}\left[\left(S S^{T}\right)^{q}\right]}$. For convenience, let us define

$$
Z_{\vec{i}}=\prod_{k=1}^{n} Z_{i_{2 k-1} i_{2 k}}
$$

Then, our semiclassical matrix model is

$$
\langle P(\vec{i}, \vec{j})\rangle_{E}=\lim _{N \rightarrow 0}\left[Z_{\vec{i}} Z_{\vec{j}}^{*}\right] G(\vec{i}),
$$

where

$$
G(\vec{i})=\left\langle e^{-M \sum_{q \geq 2} \frac{1}{2 q} \operatorname{Tr}\left[\left(S S^{T}\right)^{q}\right]} \prod_{k=1}^{2 n} R_{i_{k} i_{k}}\right\rangle_{S} .
$$

This matrix integral has, by construction, exactly the same diagrammatic formulation as the semiclassical approach.

\section{Equivalence with RMT}

We wish to show equivalence between semiclassical theory and random matrix theory, i.e. between Eqs.(31)-(32) and Eq.(6).

We start by noticing that, because of the identity

$$
\operatorname{det}(1-A)=e^{\operatorname{Tr} \log (1-A)}=e^{-\sum_{q \geq 1} \frac{1}{q} \operatorname{Tr}\left(A^{q}\right)},
$$

we can write

$$
G(\vec{i})=\int \frac{d S}{\mathcal{Z}} \operatorname{det}\left(1-S S^{T}\right)^{M / 2} \prod_{k=1}^{2 n} R_{i_{k} i_{k}} .
$$

Next, from the theory of truncated orthogonal matrices it can be established (see Appendix) that

$$
\lim _{N \rightarrow 0} \int \frac{d S}{\mathcal{Z}} \operatorname{det}\left(1-S S^{T}\right)^{M / 2} \prod_{k=1}^{2 n} S_{a_{k} b_{k}}=\left\langle\prod_{k=1}^{2 n} O_{a_{k} b_{k}}\right\rangle_{\mathcal{O}(M+1)},
$$

provided $1 \leq a_{k}, b_{k} \leq M$ and where the average on the right-hand-side is over random matrices uniformly distributed (with respect to Haar measure) in the orthogonal group of $(M+1)$-dimensional real matrices satisfying $O O^{T}=1$.

It is known [48, 49] that this orthogonal group average is given by a double sum over the set of matchings,

$$
\left\langle\prod_{k=1}^{2 n} O_{a_{k} b_{k}}\right\rangle_{\mathcal{O}(M+1)}=\sum_{\sigma, \tau \in \mathfrak{M}_{n}} \Delta_{\sigma}(a) \Delta_{\tau}(b) \mathcal{W}_{M+1}\left(\sigma^{-1} \tau\right),
$$

where the quantity $\mathcal{W}_{M+1}$ is the Weingarten function of $\mathcal{O}(M+1)$ and

$$
\Delta_{\sigma}(a)=\prod_{k=1}^{n} \delta_{a_{\sigma(2 k-1)}, a_{\sigma(2 k)}} .
$$

Noticing that

$$
\sum_{a_{1}, \ldots a_{2 n}=1}^{M} \Delta_{\sigma}(a) \prod_{k=1}^{2 n} X_{i_{k} a_{k}}=\prod_{k=1}^{n}\left(X X^{T}\right)_{i_{\sigma(2 k-1)}, i_{\sigma(2 k)}}=Z_{\sigma(\vec{i})}
$$


Semiclassical matrix model for quantum chaotic transport with time-reversal symmetry 10

leads to

$$
\lim _{N \rightarrow 0} G(\vec{i})=\sum_{\sigma, \tau \in \mathfrak{M}_{n}} \mathcal{W}_{M+1}\left(\sigma^{-1} \tau\right) Z_{\sigma(\vec{i})} Z_{\tau(\vec{i})}^{*}
$$

We now extract the coefficient of $\left[Z_{\vec{i}} Z_{\vec{j}}^{*}\right]$. The only matching $\sigma$ which survives this operation is the identity. On the other hand, we have already seen that

$$
\left[Z_{\vec{j}}^{*}\right] Z_{\tau(\vec{i})}^{*}=\sum_{\rho \in \mathfrak{H}_{n}} \delta_{\rho}(\tau(\vec{i}), \vec{j}) \text {. }
$$

Thus, we get

$$
\lim _{N \rightarrow 0}\left[Z_{\vec{i}} Z_{\vec{j}}^{*}\right] G(\vec{i})=\sum_{\tau \in \mathfrak{M}_{n}} \sum_{\rho \in \mathfrak{H}_{n}} \mathcal{W}_{M+1}(\tau) \delta_{\rho}(\tau(\vec{i}), \vec{j}) .
$$

We may use the invariance of the Weingarten function $\mathcal{W}$ under the action of the hyperoctahedral, $\mathcal{W}_{M+1}(\rho \tau)=\mathcal{W}_{M+1}(\tau)$, to group both sums into a single sum over the whole permutation group. Defining $\pi=\rho \tau$, we have

$$
\langle P(\vec{i}, \vec{j})\rangle_{E}=\sum_{\pi \in \mathfrak{S}_{n}} \mathcal{W}_{M+1}(\pi) \delta_{\pi}(\vec{i}, \vec{j})
$$

It was shown in [41] that the Weingarten functions of the orthogonal group and of the COE are related by the simple identity

$$
\mathcal{W}_{M+1}(\pi)=\mathrm{W}_{M}(\pi) \text {. }
$$

We are hereby showing that this equality, rooted in the fact that $\operatorname{COE}(M)$ can be seen as the quotient space $\mathcal{U}(M) / \mathcal{O}(M)$, where $\mathcal{U}(M)$ is the unitary group, is in fact the key to the equivalence between the semiclassical and RMT approaches to quantum chaotic transport in the presence of time-reversal symmetry, because it leads precisely to the fact that

$$
\langle P(\vec{i}, \vec{j})\rangle_{E}=\langle P(\vec{i}, \vec{j})\rangle_{\mathrm{COE}(M)} .
$$

\section{Conclusions}

We have extended the matrix model approach to semiclassical quantum chaotic transport in order to treat systems with time-reversal symmetry. The hardest part of the method is designing a matrix integral with the correct semiclassical diagrammatic rules. Once this is in place, it leads in a very direct way to the equivalence to random matrix theory.

This approach may open the way to semiclassical calculations that were previously unavailable, and may even provide results beyond RMT. For example, it may be adapted to treat problems where the semiclassical trajectories have different energies, as is necessary in calculations involving time delay (this program has already been carried out for broken time reversal symmetry in [14]). Another possibility is the treatment of the proximity gap in Andreev billiards [50].

Similar ideas might also be applied to closed systems, allowing the calculation of spectral correlation functions and providing justification for the celebrated BohigasGiannoni-Schmit conjecture [51, 52] that they are all described by RMT. 
Semiclassical matrix model for quantum chaotic transport with time-reversal symmetry 11

\section{Acknowledgments}

Gregory Berkolaiko was involved in the initial stages of this project, and interesting conversations with him are gratefully acknowledged, as well as financial support from Conselho Nacional de Desenvolvimento Científico e Tecnológico (CNPq).

\section{Appendix}

Consider a $(M+1)$-dimensional orthogonal matrix $O$, and let $S$ be its $N$-dimensional upper-left square corner. It was shown in 53 that, when $O$ is uniformly distributed in the orthogonal group $\mathcal{O}(M+1)$ with Haar measure, $S$ becomes a random matrix whose distribution is given by $P(S)=\frac{1}{\mathcal{Z}_{T}} \operatorname{det}\left(1-S S^{T}\right)^{M / 2-N}$, where the normalization constant is $\mathcal{Z}_{T}=\int d S \operatorname{det}\left(1-S S^{T}\right)^{M / 2-N}$.

Suppose we wish to compute the average value of $\prod_{k=1}^{2 n} O_{a_{k} b_{k}}$, with $1 \leq a_{k}, b_{k} \leq$ $N$. Since all the elements belong to $S$, it is obvious that

$$
\left\langle\prod_{k=1}^{2 n} O_{a_{k} b_{k}}\right\rangle_{\mathcal{O}(M+1)}=\int \frac{d S}{\mathcal{Z}_{T}} \operatorname{det}\left(1-S S^{T}\right)^{M / 2-N} \prod_{k=1}^{2 n} S_{a_{k} b_{k}} .
$$

This is an exact relation between the statistics properties of orthogonal matrices and those of general real matrices.

Introduce now the different normalization constant $\mathcal{Z}=\int d S e^{-\frac{M}{2} \operatorname{Tr} S S^{T}}$, which is the one required in the use of Wick's rule. As discussed in the text, we wish to compute the limit as $N \rightarrow 0$ of Eq.(45), in the form

$$
\lim _{N \rightarrow 0} \frac{\mathcal{Z}}{\mathcal{Z}_{T}} \int \frac{d S}{\mathcal{Z}} \operatorname{det}\left(1-S S^{T}\right)^{M / 2-N} \prod_{k=1}^{2 n} S_{a_{k} b_{k}} .
$$

It is not difficult to show that $\lim _{N \rightarrow 0} \frac{\mathcal{Z}}{\mathcal{Z}_{T}}=1$, and we therefore arrive at Eq.(35).

\section{References}

[1] R. Blümel and U. Smilansky, Phys. Rev. Lett. 60477 (1988); ibid 64, 241 (1990).

[2] H.U. Baranger and P.A. Mello, Phys. Rev. Lett. 73, 142 (1994).

[3] R.A. Jalabert, J.L. Pichard, and C.W.J. Beenakker, Europhys. Lett. 27, 255 (1994).

[4] C.W.J. Beenakker, Rev. Mod. Phys. 69, 731 (1997).

[5] F. Mezzadri, N. Simm, J. Math. Phys. 52, 103511 (2011); ibid 53, 053504 (2012).

[6] M. Novaes, Phys. Rev. B 78, 035337 (2008).

[7] B.A. Khoruzhenko, D.V. Savin, and H.J. Sommers, Phys. Rev. B 80, 125301 (2009).

[8] P. Vivo, S.N. Majumdar, and O. Bohigas, Phys. Rev. Lett. 101, 216809 (2008).

[9] C.A. Souza-Filho, A.F. Macedo-Junior, A.M.S. Macêdo, J. Phys. A 47, 105102 (2014).

[10] X. Zheng, S. Hemmady, T.M. Antonsen Jr., S.M. Anlage, and E. Ott, Phys. Rev. E 73, 046208 (2006).

[11] Y.V. Fyodorov and H.-J. Sommers, J. Math. Phys. 38, 1918 (1997).

[12] P.W. Brouwer, K.M. Frahm and C.W.J. Beenakker, Phys. Rev. Lett. 78, 4737 (1997).

[13] J. Kuipers, D.V. Savin and M. Sieber, New J. Phys. 16123018 (2014).

[14] M. Novaes, arXiv preprint arXiv:1408.1669.

[15] C.W.J. Beenakker, Phys. Rev. B 47, 15763 (1993).

[16] A. Altland and M. Zirnbauer, Phys. Rev. Lett. 76, 3420 (1996).

[17] P. A. Mello and H. U. Baranger, Waves Random Media 9, 105 (1999).

[18] P.W. Brouwer, Phys. Rev. B 51, 16878 (1995).

[19] J. G. G. S. Ramos, A. L. R. Barbosa and A.M.S. Macêdo, Phys. Rev. B 78, 235305 (2008).

[20] P. Vidal and E. Kanzieper, Phys. Rev. Lett. 108, 206806 (2012).

[21] S. Rodríguez-Pérez, R. Marino, M. Novaes and P. Vivo, Phys. Rev. E 88, 052912 (2013). 
Semiclassical matrix model for quantum chaotic transport with time-reversal symmetry 12

[22] J. Wurm, A. Rycerz, İ. Adagideli, M. Wimmer, K. Richter and H.U. Baranger, Phys. Rev. Lett. 102, 056806 (2009).

[23] A. Ossipov, M. Titov, and C.W.J. Beenakker, Phys. Rev. B 75, 241401(R) (2007).

[24] W.H. Miller, Adv. Chem. Phys. 30, 77 (1975).

[25] H.U. Baranger, R. Jalabert and A.D. Stone, Chaos 3, 665 (1993).

[26] K. Richter and M. Sieber, Phys. Rev. Lett. 89, 206801 (2002).

[27] H. Schanz, M. Puhlmann and T. Geisel, Phys. Rev. Lett. 91, 134101 (2003).

[28] S. Heusler, S. Müller, P. Braun and F. Haake, Phys. Rev. Lett. 96, 066804 (2006).

[29] P. Braun, S. Heusler, S. Müller and F. Haake, J. Phys. A 39, L159 (2006).

[30] S. Müller, S. Heusler, P. Braun and F. Haake, New J. Phys. 9, 12 (2007).

[31] G. Berkolaiko, J.M. Harrison and M. Novaes, J. Phys. A 41, 365102 (2008).

[32] G. Berkolaiko and J. Kuipers, New J. Phys. 13, 063020 (2011).

[33] M. Novaes, Europhys. Lett. 98, 20006 (2012).

[34] M. Novaes, J. Phys. A 46, 095101 (2013).

[35] G. Berkolaiko and J. Kuipers, Phys. Rev. E 85, 045201 (2012).

[36] G. Berkolaiko and J. Kuipers, J. Math. Phys. 54, 112103 (2013), ibid 54, 123505 (2013).

[37] S. Rahav and P.W. Brouwer, Phys. Rev. Lett. 95, 056806 (2005).

[38] R.S. Whitney and Ph. Jacquod, Phys. Rev. Lett. 96, 206804 (2006).

[39] D. Waltner, J. Kuipers and K. Richter, Phys. Rev. B 83, 195315 (2011).

[40] M. Novaes, J. Phys. A 46, 502002 (2013).

[41] S. Matsumoto, Random Matrices: Theory Appl. 1, 1250005 (2012).

[42] P.W. Brouwer and C.W.J. Beenakker, J. Math. Phys. 37, 4904 (1996).

[43] J. Ginibre, J. Math. Phys. 6, 440 (1965).

[44] A. Zvonkin, Math. Comput. Modelling 26, 281 (1997).

[45] P. Di Francesco, Nucl. Phys. B 648, 461 (2002).

[46] E. Brezin and H. Neuberger, Nucl. Phys. B 350, 513 (1991).

[47] G.R. Harris and M.J. Martinec, Phys. Lett. B 245, 384 (1990).

[48] B. Collins and P. Śniady, Comm. Math. Phys. 264, 773 (2006).

[49] B. Collins and S. Matsumoto, J. Math. Phys. 50, 113516 (2009).

[50] J. Kuipers, D. Waltner, C. Petitjean, G. Berkolaiko, K. Richter, Phys. Rev. Lett. 104, 027001 (2010).

[51] O. Bohigas, M.J. Giannoni and C. Schmit, Phys. Rev. Lett. 52, 1 (1984).

[52] S. Müller, S. Heusler, P. Braun, F. Haake, and A. Altland, Phys. Rev. E 72, 046207 (2005).

[53] B.A. Khoruzhenko, H.-J. Sommers, and K. Życzkowski, Phys. Rev. E 82, 040106(R) (2010). 